\title{
Paradigmas socio-educativos. Una síntesis referencial para un modelo educativo basado en la teoría de la complejidad
}

\section{Socio-educational paradigm. Referential synthesis for an educational model based on the complexity theory}

Estudios

\author{
Yolimar Padilla \\ Universidad Bolivariana de Venezuela \\ yolimarpadilla@gmail.com \\ Jorge Acosta \\ Universidad del Zulia- Maracaibo \\ jorgesabhib@gmail.com \\ Diana Perozo \\ Universidad del Zulia- Maracaibo \\ dianabperozoa@yahoo.es
}

Recibido: 16 de octubre de 2015 / Aceptado: 31 de mayo de 2016

\section{Resumen}

El objetivo es analizar los paradigmas socioeducativos que tradicionalmente han impactado la educación, a los fines de sistematizar esta información como base teórica y epistemológica para futuras investigaciones en el área. El proceso metodológico implicó tres pasos: I. Investigación documental, 2. Lectura crítica de las corrientes principales. 3. Sistematización del recorrido histórico por décadas de las teorías socioeducativas. Se finaliza incluyendo elementos definitorios de la episteme de la complejidad como un aporte a ese recuento histórico, concluyéndose que esta noción da sustento a la educación venezolana hoy y a la forja del nuevo ser humano del siglo $X X \mid$

Palabras clave: Paradigmas socio-educativos, epistemología, teoría de la complejidad.

\begin{abstract}
The objective is to analyze the socio-educational paradigms that have traditionally impacted education, in order to systematize this information as a theoretical basis and epistemology for future research in the area. The methodology involved three steps: I. Documentary Research. 2. Critical reading of the mainstream. 3. Systematic historical journey through decades of socio-educational theories. The conclusions include defining elements of episteme complexity as a contribution to the historical account, and explaining of that notion that sustains venezuelan education today and forging new human being of the XXI Century.

Keywords: Socio-educational paradigms, epistemology, complexity theory.
\end{abstract}

Forma sugerida de citar: Padilla Yolimar, Acosta Jorge, Perozo Diana (2016). Paradigmas socio-educativos. Una síntesis referencial para un modelo educativo basado en la teoría de la complejidad. Alteridad, 11(1), pp. 88-100. 


\section{Introducción}

En la primera década del siglo XXI, Venezuela experimenta un proceso de transformación, político, económico, social y educativo que demanda una obligada reflexión y revisión sobre los elementos esenciales que definen el papel de la educación en la sociedad; es decir, en cuanto a su participación activa de formación de ciudadanos comprometidos con las transformaciones que se están generado en la esfera pública. Así, desde el Estado Docente, se prevé un modelo educativo distinto. En consecuencia, tiene vigencia analizar los paradigmas socioeducativos que tradicionalmente han impactado la educación en Venezuela. El propósito, es participar y aportar en esta discusión académica permanente; de manera tal que, se pueda sistematizar esta información como base teórica para futuras investigaciones, o para nuevas propuestas educativas, con visión epistémica de alcance holista, ecológico y compromiso social, en correspondencia con el modelo de país que se gesta en Venezuela en la primera década del siglo XXI.

El proceso metodológico para llegar a estas reflexiones tiene tres pasos: 1 . Investigación documental, 2. Lectura crítica de los "paradigmas socioeducativos" propuestos por sus teóricos o representantes. 3. Sistematización del recorrido histórico de las teorías socio-educativas, por períodos: 1 er período, años 50, inicio de los 60, Teorías Tradicionales. En el 2do período, Teorías Críticas de los años 60 y 70. 3er período, años 80 , Teorías de la posición analítica. 4to reciente período desde la Teoría de la Complejidad, inicios del siglo XXI. Se finaliza haciendo un aporte a ese recuento histórico incluyendo elementos definitorios de la episteme de la complejidad, concluyéndose que la educación venezolana hoy, toma importantes aspectos de esta teoría. Se organizan aquí una serie de criterios, proponiendo desde el enfoque epistémico de la complejidad, el cual reivindica principalmente, una visión cósmica, ecológica, inclusiva de todas las culturas, de los géneros, y las especies, como sustento de la educación necesaria para el nuevo ser humano del siglo XXI.

\section{Desarrollo}

\section{Teorías socio-educativas. Breve recorrido histórico}

1er Período: Teorías Tradicionales.

Años 50 e inicio de los 60

Según Matus (2005), estas teorías surgen a mediados del siglo XIX y buena parte del siglo XX, (década de los cincuenta), período llamado también "La edad de la inocencia".

Se consideraba la educación como uno de los principales elementos para superar las injusticias sociales. De estas teorías, surge el primer período de la investigación social educativa aupada fundamentalmente, por la urgencia de integración social que se vivía en los Estados Unidos de América y a la necesidad de reconstrucción económica que se reclamaba en el continente europeo. Estas teorías fueron fundamentalmente: la Teoría Estructural-Funcionalista, la Teoría del Capital Humano y, la teoría del Empirismo Metodológico, las cuales pueden definirse así:

\section{Teoría Estructural-Funcionalista}

Su base está en la teoría positivista y organicista de Spencer y la teoría de la Acción Social de Max Weber, principalmente. También se le denomina Funcionalismo estructural o estructuralista y es empleado en ciertas ciencias sociales, en especial en la Sociología, la Antropología y la Psicología. Los principales exponentes son Emile Durkheim, Talcott Parsons y Robert Merton y sus aplicaciones tienen lugar en el campo de la sociología cultural. Esta teoría dominó en Estados Unidos, desde 1950 hasta la década de 1980, pero su impacto en el estudio científico de las Ciencias Sociales, se deja ver hasta hoy en toda América. Talcott Parsons (1967) desarrolló el funcionalismo estructural, para cuya comprensión es indispensable su esquema de nombre: AGIL; siglas que corresponden a lo que él considera cuatro imperativos funcionales en todo sistema y en la "sociedad en general": 
Cuadro 1. Modelo Global de Análisis Sistémico de la Sociedad. Paradigma AGIL

\begin{tabular}{|c|c|c|c|}
\hline Subsistema & Función & Imperativos funcionales(AGIL) & $\begin{array}{l}\text { Subsistemas en la } \\
\text { Sociedad (AGIL) }\end{array}$ \\
\hline $\begin{array}{l}\text { Biológico } \\
\text { (analogía entre } \\
\text { la vida orgánica } \\
\text { y la vida social) }\end{array}$ & Adaptación & $\begin{array}{l}\text { A: Adaptación. Todo sistema debe } \\
\text { abarcar las situaciones externas. Debe } \\
\text { adaptarse a su entorno y adaptar el en- } \\
\text { torno a sus necesidades. }\end{array}$ & $\begin{array}{l}\text { A: La economía; Adapta la } \\
\text { sociedad al entorno median- } \\
\text { te el trabajo, producción y } \\
\text { distribución. }\end{array}$ \\
\hline $\begin{array}{l}\text { Cultural } \\
\text { (normas, valores, } \\
\text { lenguaje y símbolos } \\
\text { compartidos) }\end{array}$ & $\begin{array}{l}\text { Mantenimiento } \\
\text { de pautas para } \\
\text { ajustarse a } \\
\text { expectativas }\end{array}$ & $\begin{array}{l}\text { G: (Goal Attainment). Capacidad para } \\
\text { alcanzar metas; sistema debe definir y } \\
\text { alcanzar sus metas fundamentales. }\end{array}$ & $\begin{array}{l}\text { G: La Política; busca el logro } \\
\text { de metas. }\end{array}$ \\
\hline $\begin{array}{l}\text { Social } \\
\text { (formas de interacción } \\
\text { recíproca) }\end{array}$ & Integración & $\begin{array}{l}\text { I: Integración. El sistema debe regular } \\
\text { la integración entre sus componentes y } \\
\text { entre los otros imperativos funcionales: } \\
\text { A,G y L. }\end{array}$ & $\begin{array}{l}\text { I: La comunidad societal; In- } \\
\text { tegra la sociedad como un } \\
\text { todo. }\end{array}$ \\
\hline $\begin{array}{l}\text { Personalidad } \\
\text { (motivaciones } \\
\text { y orientaciones } \\
\text { de la acción) }\end{array}$ & $\begin{array}{l}\text { Logro de metas } \\
\text { o fines personales }\end{array}$ & $\begin{array}{l}\text { L: Latencia. (Mantenimiento de Patro- } \\
\text { nes). Un sistema debe mantener, pro- } \\
\text { porcionar y renovar la motivación de } \\
\text { los individuos y las pautas culturales } \\
\text { que lo integran. }\end{array}$ & $\begin{array}{l}\text { L: El sistema fiduciario; } \\
\text { transmite culturas, normas y } \\
\text { valores. }\end{array}$ \\
\hline
\end{tabular}

Fuente: Autoría propia basada en Talcott Parsons (1967).

Sus principales postulados para explicar el desarrollo de lo social son:

- Establece una analogía tomando como modelo las funciones de los organismos biológicos para aplicar a los organismos sociales. Es ser humano es solo un individuo.

- Las funciones de grupos sociales tienden a ser más especializadas, interdependientes y eficaces mientras se desarrolla en niveles más complejos de organización.

- Las acciones o funciones conscientes e inconscientes de los individuos provocan una modalidad y función de la sociedad; esto es, depende de las características del estado social, la cultura imperante y el grupo social al que pertenece.

El sistema social tiende al equilibrio y a la estabilidad, y sus únicas fuerzas perturbadoras son los individuos; porque los conflictos se originan en la ideología de los hombres y mujeres. Los funcionalistas conciben el Estado como una organización de control, para lograr el equilibrio y administración que beneficia a toda la sociedad.

\section{Teoría del Capital Humano}

Desde Adam Smith ya se había citado la relación entre la riqueza y el trabajo de los individuos; durante la década de los años 60, este argumento fue refinado en el resto del mundo a partir del estudio sociológico realizado por Theodore Schultz y Gary Becker, dando lugar a la teoría del Capital Humano. Aunque con diferencias, entendían la educación para el provecho material, o de capital, considerándole una inversión susceptible de cálculo sobre su rentabilidad (Garrido, 2007). En consecuencia, esta teoría considera que sobre la base de las necesidades del sector productivo y de los requerimientos para el desarrollo económico-social de la Nación, se deben planificar un modelo educativo que cubra esos conocimientos, capacidades y habilidades. Este enfoque introdujo en la política económica, la noción de "inversión en capital humano", que supone, una mejora en la calidad del factor trabajo y su técnica para contribuir al crecimiento económico.

Así entonces, al llevar esta noción al plano de la educación, más específicamente a la "fun- 
cionalidad técnica de la educación", la Teoría del Capital Humano se utiliza para designar al conjunto de conocimientos, habilidades, destrezas y talentos que posee una persona y la hacen apta para desarrollar actividades específicas; lo cual, propicia el crecimiento económico. Un análisis crítico de este enfoque radica en el énfasis que le da al beneficio monetario como finalidad, en el vínculo entre la educación, la productividad y la elevada consideración a la potencialidad económica del individuo en formación y no como medio para un desarrollo social. (Fernández, 1987). Las refutaciones a esta teoría se centraron en el descuido de la relación entre éxito escolar y origen social. También, esta niega el carácter colectivo del proceso de acumulación de conocimiento y trata al individuo como mera herramienta del crecimiento económico.

\section{Teoría del Empirismo o Individualismo Metodológico}

Se basa en la lógica empírica para el planteamiento y desarrollo de la investigación científica, muy usado en el campo de las ciencias sociales y en las ciencias descriptivas. El empirismo plantea que la experiencia que dan los sentidos es la fuente de todo conocimiento. Para garantizar la cientificidad se proponen la comprobación empírica, otorgándole un fundamento abstracto, teorético, no sensible. Los representantes principales son Boudon (1979) y Coleman (1988), con su modelo de educación y movilidad social (Rincón, 1993).

Coleman propone la idea de la "elección racional", afirmando que las personas actúan intencionadamente en persecución de una meta y para alcanzarla, utilizan unos recursos que define como todas las cosas sobre las que el actor tiene un control. Una parte importante de su investigación en educación, estuvo centrada en los mecanismos y reglas para moverse desde la elección individual a la elección colectiva (social). Para ello, desarrolló el concepto de "actor corporativo", referido a los actores, no individuales, que persiguen los intereses de una colectividad (por ejemplo las asocia- ciones). En este periodo, la formación se observa dentro de un esquema económico, al mejorar los niveles educativos poblacionales se prevé un mayor potencial en su capital humano que se correlaciona con el desarrollo (Matus, 2005).

Segundo Período. Teorías Críticas. Años 60 y 70

Ya entrada la década de los sesenta, surgieron las teorías científicas con enfoque crítico, entre las cuales resaltan las teorías educativas influenciadas por el Marxismo; aun cuando, unas estuvieron sustentadas en la Psicología, otras en la Economía y un tercer grupo en la Sociología. Estas teorías, según Giroux, (1992), fueron: la Nueva Sociología de la Educación, Teorías Críticas, Teoría del Conflicto, Teoría de la Educación para la libertad.

\section{Teoría de la Nueva Sociología de la Educación (Gran Bretaña)}

Esta teoría desarrolló dos vertientes: La de la reproducción cultural y la vertiente marxista. La vertiente de la reproducción cultural, fue trabajada fundamentalmente por Basil Bernstein (1990), sociólogo y lingüista británico, con su análisis de los "Códigos lingüísticos", desde donde dialoga permanentemente con Pierre Bourdieu (1994) y sus teorías del "Capital cultural". Sus principales aportes se orientan al desarrollo de una teoría sociológica de la educación, inspirada en los trabajos de Durkheim, Weber, Marx y de los estructuralistas. Para esta corriente, el principal problema es comprender cómo una sociedad determina, selecciona, clasifica, asigna, transmite, evalúa el conocimiento, y es sometido al poder diferencial de las clases sociales y al aparato de control social de la sociedad. La característica teórica es su multidisciplinaridad, al conjugar aportes teóricos desde la antropología, sociología, psicología y lingüística.

Para Bernstein, la concepción entre proceso de socialización y código lingüístico ha sostenido que niños de diversa extracción desarrollan dos códigos lingüísticos diferentes, asociados a las pautas socializantes vigentes en familias de dis- 


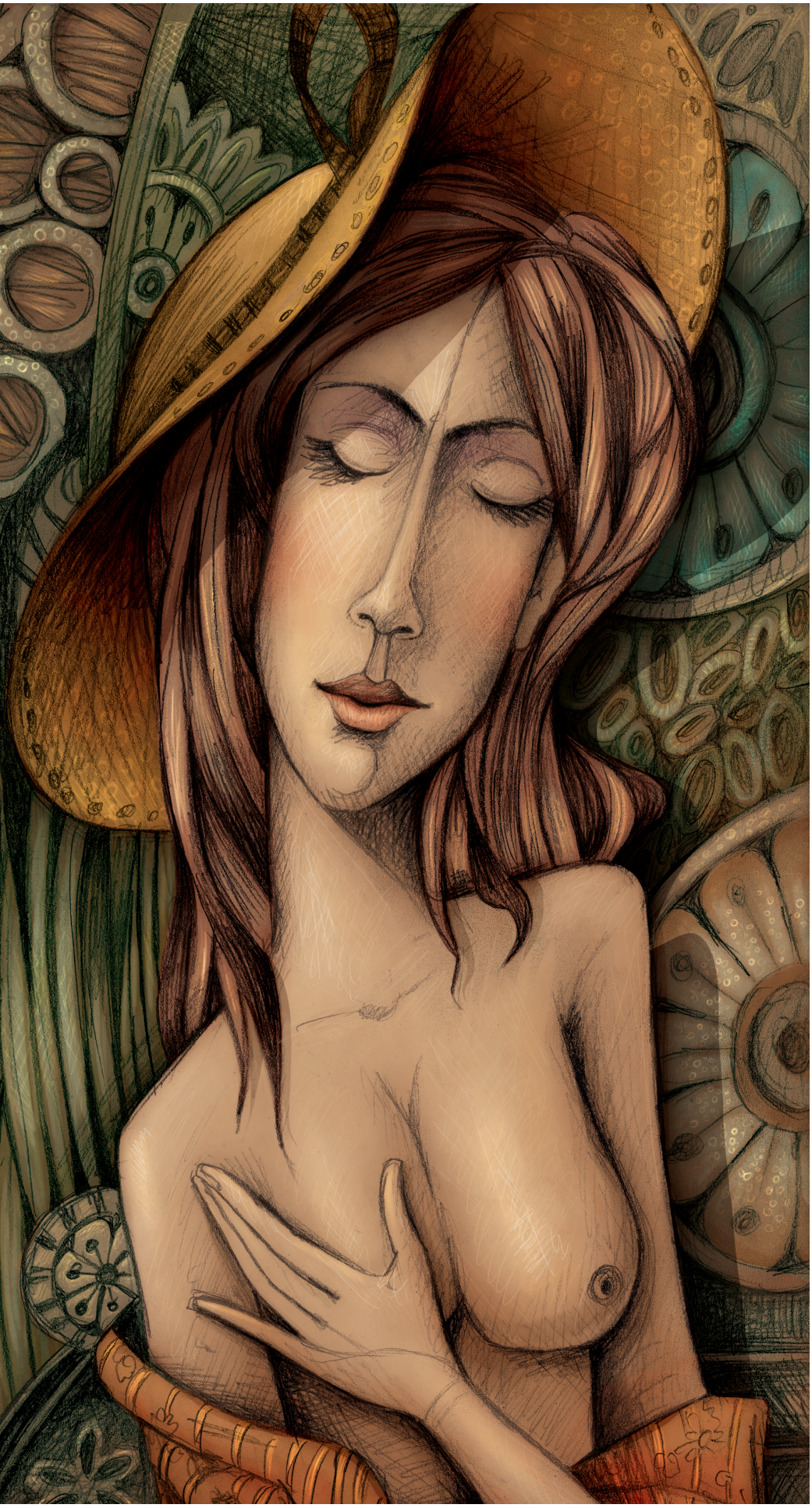

TÓCATE.

Mixta/ Lápiz sobre papel y

retoque digital tinto origen social. Planteando que ésta se puede realizar a través de tres sistemas de mensaje: el currículo, la pedagogía y la evaluación.

La segunda vertiente es de enfoque marxista. Esta es desarrollada fundamentalmente, en la sociología de la educación de Althusser (1974), cuando define la Escuela como Aparato Ideológico del Estado, que contribuye a la reproducción social de la sociedad, en la cual se va inculcando la ideología dominante a través de la transmisión cultural y clasificando los tipos de profesionales según su procedencia de clase social.

\section{Teoría de la Reflexión Crítica (Francia-Alemania)}

En este enfoque crítico se inscribe Habermas (1987) de la escuela de Viena; Carr y Kemmis (1988), de Alemania y Francia, quienes asumen la Teoría Crítica y Pierre Bourdieu (1994) de Francia, quien destaca especialmente por su labor crítica de la cultura, mostrando que la distinción cultural no es más que dominación encubierta, complicidad ontológica. Bourdieu, sociólogo francés, (1994), explica cómo los diferentes estudiantes tienen su cultura, aprendiendo, unos más que otros en la escuela y que ésta enseña la cultura capitalista. Plantea la existencia de una "violencia simbólica", afirma que la escuela transmite una cultura que no coincide con la de muchos estudiantes. Según el autor, existen diferentes tipos de capital dentro del mapa social: económico, cultural, escolar, social (relaciones sociales). Cada clase social tiene sus costumbres y gustos; es decir su cultura. Analiza, con perspectiva marxista, cómo las clases alta y media pugnan e intentan sobresalir; mientras, la clase baja y media baja, anima a sus hijos a estudiar para mejorar socialmente imitando la clase alta.

Por otra parte, la Teoría Crítica que difunde la Escuela de Frankfurt (Theodor Adorno, Erich Fromm, Max Horkheimer, Herbert Marcuse, entre otros), centra su crítica a la educación tradicional, basándose en los siguientes supuestos: la razón es reemplazada por la técnica; el pensamiento crítico es reemplazado por las normas científicas; la cien- 
cia es convertida en ideología; prevalece la contemplación del mundo sin examinarlo; su línea de acción está en el siguiente orden: valores, juicios e intereses de la humanidad; hay un desarrollo del cientificismo: (Fe en la ciencia misma); creencia positiva en la unidad lógica y metodológica de las Ciencias Sociales y Naturales; hay intereses constitutivos del saber según las necesidades naturales.

Especialmente Habermas (1984), en su teoría crítica de la ciencia, expone que existe una pretendida neutralidad científica, por lo cual se llega a una pseudo-neutralidad, como toda pretendida objetividad cognoscitiva es simplemente una ilusión, pretensiones y actitudes que una ciencia social crítica debería hacer conscientes y superar. Según Machado (2000) la postura de Habermas, establece nexos entre la crítica en la ciencia social y su implicación en el área moral, para abordar la solución de los problemas que surgen de la interacción entre los individuos, mediante el análisis crítico del conocimiento. Habermas extiende su Teoría de la Acción Comunicativa, tomando la comunicación como eje del proceso educativo, ofreciendo la siguiente categorización:

\begin{tabular}{|c|c|c|c|}
\hline Intereses & Saber & Medio & Ciencia \\
\hline Técnico & $\begin{array}{c}\text { Instrumental } \\
\text { Explicación social }\end{array}$ & El trabajo & Las empíricas, analíticas o naturales \\
\hline Práctico & $\begin{array}{c}\text { Práctico } \\
\text { Entendimiento }\end{array}$ & El lenguaje & Las Interpretativas "Hermenéuticas" \\
\hline Emancipatorio & Crítico y Reflexivo & El poder & Las ciencias críticas \\
\hline
\end{tabular}

Fuente: Autoría propia, basada en Habermas (1984)

Se interpreta en consecuencia, que en este enfoque la teoría y la práctica, son dos procesos interdependientes; al modo del concepto de "praxis" marxista. Una teoría social crítica parte de los problemas de la vida cotidiana y se construye para solucionarlos (Carr y Kemmis, 1988).

Según este enfoque, en el sujeto se presenta la necesidad de investigar, de ser intérprete de su realidad; pero, debe ser consciente respecto a que, para la solución de problemas no basta con interpretar, sino que hay que transformar. La Educación es concebida como un proceso de transmisión cultural, que esclarece la naturaleza del proceso de reproducción de las clases sociales; o sea, el proceso de explotación que se da en cada sistema para la adquisición de bienes y servicios en el área económica; así como también, en lo educativo y cultural.

Teoría del Conflicto

(Estados Unidos, 1950-1960)

Es considerada como una propuesta emergente ante el agotamiento del funcionalismo estructural para la explicación de la complejidad social; aun cuando, ésta también fue superada por las teorías neomarxistas. Esta teoría tiene varias corrientes: a) La que desarrolla la Teoría de la Correspondencia, de Samuel Bowles y Herbert Gintis, economistas, quienes en su libro Schooling in Capitalism America (1985), analizan el entorno institucional en el sistema escolar moderno. b) La denominada Escuela Clásica, del siglo XX, que se enfoca en el nivel macro, y tiende a analizar gran número de variables para comprender un conflicto: las relaciones conscientes entre grupos sociales, la visión del individuo como un actor racional y el poder como ente generador de las luchas o conflictos sociales.

Según el principio de correspondencia de esta teoría, el sistema educativo y el productivo están íntimamente relacionados y mantienen semejanza. Se afirma además, que la Escuela refleja la misma estructural existente en la sociedad (la cual es desigual para ellos); así como, el currículo escolar permite y viabiliza la enseñanza de actitudes y competencias mediante una estructura autoritaria que tiene vigencia y es necesaria en el lugar del Trabajo. La Escuela hace lo mismo que el trabajo, al reproducir el sistema de clases sociales. 
A los niños se les enseñan cualidades según su clase social, sean estos hijos de capitalistas o de los obreros. Por lo que, en la escuela se desarrollan relaciones sociales que se corresponden con las relaciones sociales de producción.

Los autores afirman que la escuela ha evolucionado hacia una forma de educación "burocrática" que impide la igualdad y el desarrollo personal. Empero, la escuela suministra capacidades técnicas y sociales, ayuda a producir consciencia sobre las relaciones sociales, para desenvolverse laboralmente en un sistema de recompensas y castigos, como réplica de lo vivido en la sociedad.

La Escuela Conductista, se enfoca en el nivel micro. Su método consiste en aislar pocas variables y aplicarlas a un gran número de conflictos para comprender el papel que desempeña cada variable. Entre los supuestos fundamentales, está su preocupación central por el individuo antes que por el grupo, resaltando los factores inconscientes en la generación de los conflictos. Afirman que las raíces de la guerra se encuentran en la naturaleza del comportamiento humano.

Randal Collins (1975-1996) por su parte, desarrolla con éxito una Teoría del Conflicto con enfoque integrador; aportando una orientación micro, complementando la perspectiva macro de Dahrendorf y otros; en un intento por mostrar que la estratificación y la organización se basan en las interacciones de la vida cotidiana.

Se conoce a Collins, como el impulsor de la Teoría credencialista de la educación, en la cual están presentes los fenómenos de dominio y control social entre grupos de diversa posición, con distintos saberes y estilos de vida, que compiten por los recursos, el poder y el prestigio según los títulos académicos. Este autor, a diferencia de otros que tienen el análisis a un nivel societal, deja de lado de la perspectiva política e ideológica del conflicto y le entiende desde un punto de vista individual, dadas sus raíces teóricas en la fenomenología y la Etnometodología (Collins, 1990).

Sin embargo, que no se puede construir un análisis sin el nivel macro y/o social; contemplando estos espacios como inseparables. Mientras la mayoría de los teóricos del conflicto creen que las estructuras sociales son externas y coercitivas, Collins prefirió considerarlas como pautas de interacción y son los actores sociales quienes crean y recrean siempre la organización social.

El desarrollo de este pensamiento permitió el planteamiento de nuevas escuelas como la Teoría de la Negociación o Resolución de Conflictos y la Teoría de los Juegos de Thomas Schelling (Premio Nobel, 2005), enfoque que integra la comunicación, la información y la negociación.

\section{Teoría de la Educación \\ para la libertad (Latinoamérica)}

Esta es una tendencia multifacética, que se va desplegando en América Latina, cuya posición crítica ve la situación de "dependencia" de los pueblos, convirtiéndose en una propuesta de educación liberadora (en lo socio-político), que viene naciendo en las dos últimas décadas. Esta es protagonizada por autores latinoamericanos como Paulo Freire (brasileño), Manuela Espejo (ecuatoriana); Enrique Dussel (argentino), José Pedro Varela (uruguayo); Félix Varela y Morales (cubano), José Martí (cubano); Lázaro Cárdenas (mexicano); Pedro Henríquez Ureña (dominicano); Edelberto Torres Espinosa (nicaragüense), José Carlos Mariátegui (peruano); Aníbal Quijano (peruano); Gabriela Mistral (chilena); Fausto Reinaga (boliviano); Fals Borda (colombiano); De Sousa, Boaventura; y otros.

En Venezuela, esta corriente toma idearios de Simón Rodríguez, Andrés Bello, Simón Bolívar, Cecilio Acosta, José Pío Tamayo, Mariano Picón Salas, Luis B. Prieto Figueroa, Belén Sanjuán, Ángela Calzadillas, entre otros. Entre muchos elementos se coincide en que se deben formar hombres y mujeres útiles y productivos, moldeando un sistema pedagógico estructurado para cubrir las necesidades del actual tiempo y espacio, brindando aportes significativos al desarrollo social y el pensamiento latinoamericano. También, se asume la noción del Estado Docente el cual provee un Currículo que implica una propuesta ideológica 
para América Latina y el Caribe, planteando un proyecto propio de educación. En el desarrollo de ese proyecto deben construirse saberes científicos, pero la finalidad es "transformar".

\section{3er Período: Teorías de Posición Analítica.} (Años 80). La Interpretación Fenomenológica

Los aportes a la Teoría de la Socialización de la Educación de Young y Whitty (1977), realmente inició en la década de los 70 y tuvo su mayor auge en los 80 . Intentó comprender la vinculación entre las subjetividades y los problemas de control y poder. Estas teorías dan un giro a los estudios sobre educación orientándolos hacia la micro-sociología. Se desplaza el foco de atención de lo estructural a lo cotidianamente vivido. Se sirvieron a menudo de la llamada sociología interaccionista que permite investigar los procesos concretos de interacción entre estudiantes y profesores en el aula.

Estos autores coincidían con marxistas y weberianos en diagnosticar la desigualdad educativa y en la urgencia de plantear cambios revolucionarios en las prácticas educativas. Comenzaron a entablarse diálogos, debates, discusiones teóricas sobre el papel de la educación como reproductora de los aparatos del Estado, bajo la influencia de la escuela de Frankfurt; especialmente, de uno de sus principales exponentes: Althusser, quien explicaba el proceso de "reproducción", que se da cuando la escuela enseña ciertos tipos de saber y de saber hacer; de manera que, se asegure el sometimiento a la ideología dominante. Esto es, se enseña a saber hacer para servir y a saber controlar. De tal manera que, el grupo en el poder asegura la mano de obra con fuerza de trabajo calificada, vía la escuela y asegura la gente que tendrá bajo control a los dominados. Así entonces, el sistema obliga a los maestros en proceso de alienación a ser reproductores del sistema, ya que las escuelas representan las normas y los valores que necesita el grupo en el poder, lo que asegura las divisiones sociales y actitudes necesarias y convenientes para una división del trabajo, para la sumisión desde el hogar, la comunidad, la iglesia, etc.

\section{Teoría del Currículo Oculto. Sociología del currículum}

La expresión currículo oculto fue propuesta por Jackson Philip en su texto Vida en la clase (1968) donde afirmó que la "educación" es un proceso de socialización. Posteriormente, Benson Snyder, (1970) trabaja el concepto en su obra El currículo oculto, donde sostuvo que muchos de los conflictos intra-escolares son causados por las mismas normas sociales y académicas tácitas, que frustran las capacidades de los estudiantes para desarrollarse con independencia o para pensar creativamente. En Latinoamérica, Paulo Freire, con la Pedagogía del oprimido (1972), se ocupó del tema al explorar diferentes efectos de la enseñanza en las escuelas y el efecto de la sociedad en el proceso estudiantil.

La teoría del currículo oculto, se refiere a las formas en que una sociedad selecciona, clasifica y distribuye el conocimiento educativo, así como sus formas ocultas de dominación. Plantea que los profesores enseñan lo que quieren enseñar $y$ otras cosas ocultas que no piensan en enseñar. Y la resistencia de los estudiantes a la norma y a seguir los contenidos programáticos encuentra explicación en el currículum oculto, en contraposición está la disciplina escolar, en aras de confeccionar y comprobar el logro de objetivos de aprendizaje, mediante los cuales se logra el control del grupo, que finalmente, lleva hacia la reproducción de normas y valores sociales que persigue el grupo hegemónico y que conlleva a remarcar las distinciones entre grupos sociales.

El concepto que expresa el currículo oculto, es que los centros educativos hacen más que la simple transmisión de conocimiento que establecen los currículos oficiales, pues hay implicaciones sociales, políticos y productos culturales en las actividades educativas modernas. Varios aspectos del aprendizaje contribuyen a la difusión del currículo oculto, incluyendo: Las prácticas, los procedimientos, las reglas, las relaciones, la estructura social del aula, el ejercicio de autoridad del profesor, el lenguaje del profesor, los libros de texto, los apoyos audiovisuales, las medidas dis- 
ciplinarias, la evaluación y las prioridades curriculares. Según Henry Giroux y Anthony Penna (1992) el currículo oculto es analizado por varias teorías educativas cuya visión se ocupa del rol de los centros educativos en la socialización:

1. El punto de vista estructural-funcional de la escolarización, se centra en cómo las normas y valores son acarreados en el seno de los centros educativos y cómo sus necesidades para el funcionamiento de la sociedad devienen en su aceptación.

2. El punto de vista fenomenológico referido a la "nueva" sociología de la educación sugiere que el significado es creado a través de encuentros e interacciones situacionales, e implica que el conocimiento es de alguna manera objetivo.

3. La crítica radical neo-Marxista de la teoría y la práctica de la educación, reconoce la relación entre la reproducción y perpetuación económica y la cultural, lo cual refuerza las relaciones entre las prácticas sociales, la ideología y la teoría del aprendizaje.

\section{Teorías de la Resistencia}

\section{(Willis y Giroux)}

Esta teoría viene de la escuela de Frankfurt y desarrolla estrategias de oposición frente a la situación de dominación en el ámbito escolar, reivindicando al sujeto como agente social capaz de resistirse ante la dominación. Para Giroux (1992), la Resistencia según su acepción política, implica una noción dialéctica de la intervención humana, no como "oposición" de los oprimidos como sujetos individuales pasivos en ese proceso unilateral e individual de rebeldía; sino por el contrario, la Resistencia, se entiende como un proceso complejo de interrelación entre un grupo y las fuerzas de poder, dominación y opresión, donde, los grupos sociales, son capaces de crear y auto reflexionar, resistirse con esperanza, trascender hacia la emancipación personal y social a pesar del poder de los sectores dominantes.

Puede afirmarse que, los teóricos de la Resistencia ponen énfasis en dos temas centrales: el factor humano de los grupos sociales (agentes sociales) en pro de la emancipación es la educación y el proceso relacional entre la escuela y la sociedad dominante. La teoría de la resistencia ha intentado demostrar que, las escuelas representan el lugar donde las culturas dominantes y dominadas se enfrentan y entran en contradicción; o sea que, las escuelas no siempre son funcionales para el sistema imperante y sus intereses. También reconocen que, los campos de resistencia existen, rechazan la imposición social-cultural y reivindican la creatividad e inventiva en las clases subordinadas. Para Del Val (2001), los objetivos de la institución educativa bajo la perspectiva de la Resistencia deben ser los siguientes: a) Apoyar el desarrollo psicosocial del estudiante. b) Posibilitar el entendimiento, la explicación racional y la capacidad del estudiante para actuar sobre los fenómenos naturales y sociales. c) Ofrecer al estudiante la capacitación para comunicarse inteligentemente con los demás en su entorno social. d) Contribuir a la formación individual y social del estudiante, proporcionándole los recursos que le ubiquen en una posición autónoma y crítica, pero de relaciones positivas dentro de su grupo social.

A juicio de las autoras, respecto a las Ciencias Sociales y específicamente en las Ciencias de la Educación, es urgente profundizar en las diversas tendencias epistemológicas y analizar críticamente las perspectivas filosóficas que caracterizan la producción de conocimientos en este campo; dado que ello será el preámbulo de un cambio de paradigma. Es urgente esta profundización, para estar a la altura de los cambios científico-tecnológicos, sociales, económicos y culturales, que ha atravesado la humanidad en el transcurso de los siglos. Sobre todo en este siglo XXI, en el cual se asume que se vive en una sociedad del conocimiento, globalizada, informada y adelantada científicamente, en la cual ha surgido una nueva racionalidad, donde se integran saberes, se supera la pretensión objetivadora y dominadora de la razón técnica que se ha desarrollado hasta ahora mayormente en las ciencias, a partir de una posición post-moderna, post-estructuralista, des-contruccionista. Esta 
racionalidad es integral e integradora y reconoce diversas formas de hacer ciencia, desde diversos escenarios no necesariamente académicos; se apoya en la hermenéutica, la dialéctica y en la teoría crítica, lo cual ha generado nuevas teorías, metodologías para abordar lo social, como la Etnometodología, el Interaccionismo Simbólico, la Teoría de las Representaciones Sociales, la Teoría de la Complejidad, entre otras.

Cuarto Período: Teoría de la Complejidad. La educación para el siglo XXI

A proposición de las autoras, se plantea ubicar un cuarto periodo en estas teorías conducentes a nuevo modelo educativo. Castilla (2008), sostiene que la incapacidad de los paradigmas de la modernidad para dar respuesta a esta realidad compleja, ha ocasionado que se produzca un análisis crítico de cómo producir conocimiento, incorporándose así nuevos elementos que permitan comprender mejor la realidad. Afirma, que se requieren nuevas formas de realizar ciencia, donde se integren conocimientos, mediante experiencias dinámicas de interdisciplinariedad y transdisciplinariedad, asumiendo así un pensamiento complejo, multidimensional, que permita eliminar el paradigma de la simplicidad, el cual ha ocasionado una visión reduccionista, unidimensional, fraccionalista.

En este sentido, Morin (2002) propone "Los siete saberes necesarios para la educación del futuro", los cuales buscan contribuir a la reflexión de cómo educar para lograr un futuro sostenible. Según el autor, urge cambiar hacia otra espíteme que permita a las entidades que tienen como misión la educación, redimensionar su enfoque y romper paradigmas tradicionales, que solo han desviado al ser humano de su verdadera esencia, cósmica, ecológica y humana. En resumen plantea:

1. Enseñar sobre las cegueras del conocimiento: el error y la ilusión: Para el autor, la educación tiene como gran misión comunicar los conocimientos, pero afirma que existe suficiente documentación científica para testificar que este no se cumple; sino que por el contrario, a nivel mundial las instituciones educativas permanecen "ciegas ante lo que es el conocimiento humano, sus disposiciones, sus imperfecciones, sus dificultades, sus tendencias", creándose lo que él denomina el "error" como "la ilusión", en la conducción de los conocimientos. El estudio del conocimiento y de cómo este se obtiene, se conduce desde la epistemología y la propuesta es que esta materia debe aparecer permanente y obligatoria en todos los niveles de enseñanza. También, es importante, difundir conocimientos sobre el estudio de las características cerebrales, mentales y culturales del conocimiento humano, de sus procesos y modalidades, de las disposiciones, tanto psíquicas como culturales de los seres humanos, para tomar consciencia de sus potencialidades adormecidas.

2. Enseñar sobre los principios de un conocimiento pertinente: Este sostiene que la educación debe promover un conocimiento pertinente, que contemple "el contexto", "lo global", "lo multidimensional" y "lo complejo". Insiste sobre la necesidad de promover un conocimiento capaz de abordar problemas globales y fundamentales para inscribir allí conocimientos parciales y locales. Con esto se superará el conocimiento fragmentado por disciplina y permitirá ver las partes y las totalidades, para poder analizar los problemas desde sus contextos y sus complejidades.

3. Enseñar la condición humana: El ser humano debe ser comprendido como un ser complejo y holístico, que es a la vez físico, biológico, psíquico, cultural, social e histórico, pero la educación tradicional lo muestra parcial y de esa misma forma, busca las soluciones a sus problemáticas. Deben desarrollarse propuestas educativas integrales a partir de las cuales sea posible reconocer la unidad y la complejidad humanas y su unicidad con los demás seres de la naturaleza. 
4. Enseñar la identidad terrenal: La educación en su mirada parcial de lo humano y separada de la naturaleza, no permite a las personas tener conciencia del destino planetario del género humano por lo que debe convertirse en uno de los mayores objetivos de la educación. Es pertinente enseñar la historia de la era planetaria, los cambios geográficos, los movimientos históricos, geopolíticos, las opresiones y dominaciones de los humanos y de la naturaleza que han incidido en el planeta.

5. Enseñar a enfrentar las incertidumbres: La forma como se ha enseñado tradicionalmente la ciencia, desde el paradigma cartesiano, y el desarrollo de la modernidad, ha hecho adquirir muchas certezas, pero hoy está planteado revelar, los campos de la incertidumbre y la complejidad de la vida. Se tendrían que enseñar estrategias que permitan afrontar riesgos, lo inesperado, lo incierto y modificar su desarrollo según las informaciones adquiridas en el camino.

6. Enseñar la comprensión: Otro aspecto de un saber necesario es el valor Comprensión. A pesar de que la comprensión es al mismo tiempo medio y fin de la comunicación humana, el autor dice que aún la humanidad se encuentra en estado bárbaro de incomprensión y que este valor ha sido excluido en los ámbitos educativos en todas las edades. Esta enseñanza se centra, no sólo en los síntomas, sino en las causas del racismo, la xenofobia, las injusticias que justifican las guerras, la exclusión y la explotación de la naturaleza y del ser humano. Lo cual constituiría, una base segura para la educación por la paz, a la cual estamos ligados por esencia y vocación.

Enseñar la ética del género humano: Morin afirma (2002) que la educación debe conducir a una "antropo-ética", ya que la condición humana, es individuo-sociedad-especie. De allí, se esbozan las dos grandes finalidades ético-políticas del nuevo milenio: 1. establecer una relación de control mutuo entre la sociedad y los individuos por medio de la democracia y 2 . Concebir la humanidad como comunidad planetaria. La educación debe elevar la toma de conciencia de la Tierra-Patria.

En resumen, el pensamiento complejo permite la rearticulación de los conocimientos mediante la aplicación de principios generativos. La complejidad es lo contrario al pensamiento simple, a lo único; ya que es un tejido, heterogéneo, inseparable y asociado de lo uno y lo múltiple, del orden y el caos, de lo seguro y la incertidumbre. Se interpreta que en esta teoría de la complejidad, el ser humano es un todo bio-psico-socio-espiritual integrado que funciona a la perfección como un sistema integrado complejo, en un proceso geo-socio-histórico-sistémico-ecológico, donde todo afecta e interactúa con todo. Su lógica dialéctica implica un sistema auto-correctivo, de retro-alimentación y pro-activo que funciona a la perfección como un sistema integrado complejo, universal, interdisciplinario, transdisciplinario.

\section{Conclusiones}

El análisis de las teorías socioeducativas, permitió ubicar los diferentes paradigmas con los cuales se conduce la educación y se generan los modelos curriculares, instruccionales, didácticos vigentes pero ya agotados, lo cual conlleva a plantearse la urgencia de una ruptura epistémica en los modelos educativos en Venezuela. A partir de ese análisis, se concluye que la educación no puede ser pensada desde un solo ámbito de acción; deben tomarse en consideración: lo político, lo económico, lo social, lo ético, entre otros, lo cual permitirá abordar la realidad de forma total y particular a la vez, logrando así, la comprensión de la realidad existente. Es por ello que, las instituciones educativas deben afrontan retos complejos, lo que exhorta a que se orienten a transformaciones profundas y a ir repensando críticamente sus funciones.

Se asume en consecuencia, la necesidad de superar los enfoques tradicionales, funcionalista y positivista que se plantearon en este análisis y que priorizan una educación sólo para obtener empleo, 
para resolver las necesidades básicas materiales; por lo que, se concluye proponiendo el reconocimiento de la teorías críticas de influencia marxista/ neo-marxista y la teoría de la complejidad.

La pedagogía (en general) y además, específicamente, el sistema de educación de Venezuela necesitan un nuevo paradigma socio-educativo, precisamente en este siglo XXI, donde se habla de una sociedad del conocimiento, globalizada e informada, que da relevancia al conocimiento. Inexorablemente, emerge una nueva espíteme, una nueva racionalidad más integral e integradora de diversas formas de hacer ciencia, más dialéctica, más ecológica, más hermenéutica, más crítica y reflexiva, una racionalidad que atiende la complejidad humana desde las significaciones sociales, que transforma el modo de pensar, percibir y valorar, que reconoce que los fenómenos físico, biológicos, psicológicos, sociales, históricos, políticos y ambientales tienen de facto interconexiones múltiples recíprocamente interdependientes a nivel global.

Según Morin (2002), cada paradigma responde a una matriz epistémica que define el modo general del conocer y los significados que cada conglomerado social le asigna a las cosas, fenómenos y procesos, por lo cual, "la realidad" es una representación simbólica; así como también, la filosofía, las teorías y nociones científicas se define según el empoderamiento de una comunidad científica determinada. Como consecuencia cada disciplina debe realizar una revisión, reformulación de sus estructuras lógicas aisladas e independientes del sistema total con que necesariamente interactúa, respetando el pensamiento diverso y múltiple; donde cada elemento se defina por su red de relaciones con todos los demás. Todos estos cambios llevan a reestructurar nuevos supuestos, filosóficos, metodológicos y técnicos para el desarrollo de las ciencias.

En consecuencia, debido a que los tiempos cambian y el ser humano a la vez, el modelo de ciencia que permitió el desarrollo en otras épocas se hizo insuficiente ante los nuevos retos de la sociedad del conocimiento del siglo XXI, no solo por las implicaciones de la globalización, sino debido a la proliferación de los conocimientos, disciplinas y especialidades, lo que lleva a la búsqueda de un nuevo modelo que reconozca la integración e interconexión de las diferentes áreas del saber.

Lo que Morin (2002) ha definido como "incertidumbre" en los aspectos fundamentales del ser humano conllevó, a un cuestionamiento de los fundamentos del conocimiento científico, a una crisis del pensamiento e ineludiblemente a una nueva racionalidad científica a una "nueva ciencia" diferente al modo tradicional de pensar, mecanicista, objetivista y racionalista lógico. Por esta razón, es necesario evidenciar las contradicciones e insuficiencias de ese paradigma científico clásico que había dominado, el cual resultó corto, delimitado al objeto, determinista, con una lógica formal y verificación preestablecida desde el experto académico o científico, lo cual lo hace insuficiente e inadecuado para los procesos naturales y humanos que se imponen a lo largo de este siglo XXI; tomando en consideración que hoy día ya son reconocidas y han alcanzado un alto desarrollo nuevas ciencias, tales como las que estudian el mundo subatómico, la física cuántica, la biotecnología, la geobiología, la neuroinmunología, la neurolingüística. Esto se debe a que se están creando conceptos distintos, transdisciplinarios, interrelacionados que puedan proporcionar explicaciones globales y unificadas, en otras palabras, explicaciones complejas del cosmos.

Se cierra, abogando por que se implemente en la educación formal y popular, la nueva perspectiva de la complejidad, considerándola más completa, transdisciplinaria, holística y pertinente para la educación en el siglo XXI, basada en la sinergia entre familia-comunidad-ambiente laboral-sociedad y Estado y más acorde con las necesidades de la Venezuela de hoy.

Se comparte el enfoque de la complejidad, porque se planea mejorar el conocimiento y análisis científico, contribuir a armonizar las complejas interrelaciones entre la ciencia, la tecnología y la sociedad, partiendo de que un nuevo compromiso social de la ciencia debería basarse en la erradicación de la pobreza a partir del desarrollo sustentable y la armonía con la naturaleza-cosmos. 
En correspondencia con este modelo epistémico, el proceso educativo debe ser tratado en todas sus perspectivas: histórica, política, moral, cultural, social, y espiritual; es decir, subrayando la intencionalidad holística hacia la sociedad, donde se exprese la relación ciencia, tecnología, sociedad y esté presente el análisis cualitativo, el respeto al cosmos, a la naturaleza, a las especies, a las subjetividades, a la diversidad cultural, a las etnias, al género. Ello es garantía para ir hacia la transformación del modelo educativo en la Venezuela de hoy.

\section{Referencias bibliográficas}

AAlthusser, L. (1974). Ideología y aparatos ideológicos del Estado. En: La filosofía como arma de la revolución, (14.a ed.). México: Siglo XXI.

Bernstein, B. (1990). La construcción social del discurso pedagógico. Bogotá: Ed. Mario Díaz.

Boudon, R. (1979). La lógica de lo social. Madrid: Ed. Rialp.

Bourdieu, P. (1994). Razones prácticas. Sobre la Teoría de la Acción. Barcelona: Anagrama.

Carr, W. y Kemmis, S. (1988). Teoría crítica de la enseñanza. Barcelona: Martínez Roca.

Castilla, C. (2008). Educación popular, juventud-participación. Una alianza posible. Buenos Aires: CLACSO.

Coleman, J. (1988). Social Capital in the Creation of Human Capital, American Journal of Sociology, 94: 95-120; [ed. cast.: Capital social y creación de capital humano, Zona Abierta, 94-95 (2001): 47-81].

Collins, R (1990). Cuatro Tradiciones Sociológicas. México: UAM Iztapalapa.

Collins, R (1996). Cuatro Tradiciones Sociológicas. México: UAM Iztapalapa.

De Sousa, B. (2010). Refundación del Estado en América Latina. Perspectivas desde una epistemología del Sur. Lima: Instituto Internacional de Derecho y Sociedad.

Delval, J. (2002). La escuela posible. Barcelona: Ariel.
Fernández, A. (1987). Alcances de la explicación económica en el ámbito educativo. Revista de educación superior. México: ANUIES.

Freire, Paulo (1972). Pedagogía del oprimido. Buenos Aires: Siglo XXI.

Garrido C. (2007). La educación desde la teoría del capital humano y el otro. Revista Educere, 11(36).

Giroux, H. (1992). Teoría y resistencia en educación. México: Siglo XXI.

Giroux, H \& Penna A. (1992). Teoría y resistencia en educación. México: Siglo XXI.

Habermas, J. (1984). Ciencia y técnica como ideología. Madrid: Tecnos.

Habermas, J. (1987). Teoría de la Acción Comunicativa. (2.a ed., Vol. II). Madrid: Taurus.

Jackson, Philip (1968). Vida en la clase. Madrid: Morata.

Machado, M. (2000). Análisis crítico de la estrategia de aplicación de la teoría Crhris Argycis en su dimensión de la teoría del uso. Modelo I y Modelo II. Caracas: UCV.

Marx y Engels (1978). Textos sobre educación y enseñanza. Madrid: Comunicación.

Matus, L. (2005). Estrategias de comunicación en el ámbito rural marginado. México: UNAM.

Morin, E. (2002). Los siete saberes necesarios a la educación del futuro. CIPOST. Disponible en: http// www2.scielo.org.ve/. 20/07/2013.

Parsons, T. (1967). Clases sociales y conflictos entre clases a la luz de la reciente teoría sociológica. Buenos Aires: Paidós.

Rincón de Villalobos, B. (1993). Propuesta de aplicación del modelo investigación acción de Argyris, en la formación del rol de investigador en los docentes. Tesis de Doctorado. Tarragona: Universidad Rovira i Virgili.

Snyder, B. (1970). The hidden curriculum. New York: Knopf.

Weber, M. (1964). Economía y sociedad. (4.a ed., Vol. II). México: Fondo de Cultura Económica.

Young M, Whitty. (1977). Teoría crítica de la educación $y$ discurso en el aula. Madrid: Paidós.

Young, R. (1993). Teoría crítica de la educación y discurso en el aula. Madrid: Paidós. 